\title{
Reglas generales de acentuación en octavo grado de Educación a Distancia en la comunidad Dipina, Waslala, 2016
}

Eddlyn Antonia Granados Gómez Jiomar Antonio Marín López ${ }^{2}$ Angélica Leonor Ruíz Calderón ${ }^{3}$

\section{Resumen}

La investigación se realizó en el centro escolar "Isabel Amador", comunidad Dipina central de la Ciudad de Waslala, Región Autónoma Costa Caribe Norte (RACCN). Se ha diagnosticado la problemática que incide en la aplicación de reglas generales de acentuación en octavo grado de educación a distancia; el diseño de un plan de acción que facilite el aprendizaje, la aplicación de las reglas generales de acentuación e implementación de las estrategias metodológicas innovadoras. La población fue de 40 estudiantes más 2 investigadores y la muestra, 20 docentes. Los instrumentos utilizados fueron entrevistas y revisión documental de las diferentes estrategias implementadas: análisis y sopas de palabras, el pastel y círculos estudios. Se verificó los factores negativos que inciden en los aprendientes: falta de interés y hábitos de lectoescritura, por ende ausencia de la práctica de las reglas generales de acentuación. La población estudiantil, presentan dificultades en todos los ítems que se evaluaron y se relacionan con dificultades en la conceptualización de palabras agudas, graves, esdrújulas y sobreesdrújulas con un porcentaje de desaprobados del 90\% al inicio (diagnóstico) y una disminución hasta el 15\% al finalizar la investigación. En docentes se cotejó la ausencia del uso de estrategias innovadoras.

Palabras clave: acentuación; aprendizaje; estudiantes; estrategias; factores negativos; reglas de acentuación.

\section{Summary}

This research was carried out at the "Isabel Amador" school, located in the community of central Dipina, from the city of Waslala, Autonomous Region of the North Caribbean Coast (RACCN). The problem affecting the application of general rules of accentuation in eighth grade of distance education have been identified, which includes: the design of an action plan to facilitate learning, the application of general rules of accentuation and the implementation of innovative methodological strategies. The population was of 40 students plus 2 researchers and the sample, 20 teachers. The instruments used

\footnotetext{
Lic. Ciencias de la Educación con mención en Español. Docente rural. E-Mail: Eddlyng1o@gmail.com Lic. Ciencias de la Educación con mención en Español. Docente rural. E-Mail: iomaradirck8o@gmail.com

PhD. Educación especialidad en mediación pedagógica, Vicerrectora del Recinto las Minas. E-Mail: leoruiz_71@ Hotmail.com
} 
were interviews and documentary review of the different strategies implemented: analysis and word puzzle, pie and study circles.

We verified the negative factors that affects the students, such as lack of interest and habits of reading-writing, therefore, this causes the absence of practicing the general rules of accentuation. The student population presents difficulties in all the items that was evaluated and that are related to difficulties in the conceptualization of oxytone words, paroxytone words, proparoxytone and propoparoxytone words, and this is demonstrated with a disapproved percentage of $90 \%$ at the beginning (diagnostic) and a decrease of $15 \%$ at the end of the investigation. As for teachers, we checked the absence of the use of innovative strategies.

Keywords: accentuation; learning; students; strategies; negative factors; accentuation rules.

\section{Introducción}

Desde siempre, la enseñanza del lenguaje ha sido el tema mas importante de la escolarización del estudiantado. Esta situación no ha cambiado, lo que se modificado es el enfoque que se le da a la enseñanza de la lengua. Es imperativo, entonces, resignificar en la actualidad lo que significa la enseñanza y aprendizaje de esta área especifica.

Lengua y Literatura, es una asignatura que se prioriza por la importancia que tiene en la formación integral de las nuevas generaciones, la cual se ve obligada a una constante y profunda perfección por el papel que desempeña en la formación de los educandos, pues contribuye a la preparación para la vida laboral, económica y social, de manera que se dispongan de sólidos conocimientos lingüísticos, que les permitan interpretar avances de la ciencia y la técnica (Urrian, 1985, pág. 5).

Es vital importancia el trabajo sistemático en esta asignatura, al constituirse en un problema complejo en el aprendizaje de los escolares en todos los grados y niveles de enseñanza en Nicaragua y del mundo y a pesar del empeño de los pedagogos y lingüistas, las deficiencias continúan (Moreno, 2005, pág. 1).

Se hace la investigación para permitir un manejo del idioma, concreto al saber escribir y expresarse con soltura y desarrollar capacidades verbales a través del buen empleo de la pronunciación de las palabras, sonidos y locuciones en dicha disciplina. Los hallazgos, sirven para hacer objetivo el hábito de la aplicabilidad de reglas de acentuación. A los diferentes centros educativos, en la disciplina de Lengua y Literatura, que por falta de implementación didáctica efectiva, se presenta la problemática, así mismo contribuye con los docentes en cuanto a conocimientos teóricos y prácticos en la disciplina, mediante la implementación de nuevas estrategias metodológicas. 


\section{Revisión de literatura}

La ortografía, tiene su origen en contexto de situaciones de escritura, porque al escribir y revisar el escrito es cuando se logra la concentración en la ortografía. La ortografía comienza a codificarse en el S. XVIII, con el establecimiento en 1927 de las primeras normas ortográficas por parte de la Real Academia Española al poco tiempo de su fundación. Hasta ese momento las vacilaciones en las grafías eran constantes. El resultado era una falta de unidad que dificultaba la comprensión (Castell, 2014, pág 12).

Fuentes (2010), explica que la palabra ortografía, viene del griego orthos, que quiere decir, correcto y de orthos, que significa escribir. Así, tenemos que ortografía quiere decir escribir correctamente. Tal como debernos hacerlo cuando hablamos y al escribir, hay que tener en cuenta que existe una manera correcta para utilizar las palabras.

Actualmente, el ente regulador del idioma y la ortografía es La "Real Academia Española" (RAE), según el artículo primero de sus estatutos, tiene como misión principal, velar porque los cambios que experimente la Lengua Española en su constante adaptación a las necesidades de sus hablantes, no quiebren la esencial unidad que mantiene en todo el ámbito hispánico (Leyva, 2001, pág 6).

El aprendizaje de la ortografía, es una cuestión de percepción sensorial que sin discusión alguna, presenta múltiples y diversas dificultades. De ahí, el éxito que su enseñanza radique en el arte de desarrollar percepciones claras, completas y correctas de los vocablos que son objeto de estudio, sin que se margine su importancia desde el punto de vista psicológico, por la relación que tiene que existir entre la palabra y el objeto que ella designa (Moreno, 2005, pág3).

Enfatizando las principales dificultades que representan los estudiantes en la ortografía acentual, Calderón (2010), expone que la problemática se presenta, debido que algunos docentes no utilizan estrategias adecuadas en su labor diaria ni articulan debidamente los contenidos teóricos de la lengua y las prácticas de enseñanza. Además, que siguen prácticas tradicionales y porque toman la concepción de la escritura como producto, empleando la redacción no como una forma personal de procesar información ni como una herramienta para interactuar con el entorno donde los estudiantes presentan problemas en su comunicación escrita (p.48).

La tildación, es para muchos motivos de dudas y vacilaciones a lo largo de su formación educativa. Algunos optan por una posición defensiva y conformista llegando a decir que «cada quien escribe como le da la gana», otros creen que la computadora se constituye en la solución a sus problemas; pero quienes han tenido la oportunidad de hacer un análisis crítico de lo que han escrito, habrán podido comprobar, que quién decide es el redactor. Veamos dos situaciones: qué hacer, por ejemplo, en el caso de palabras similares que cambian de significado gracias a la posición del acento como 
hábito, habito, habitó, aquí quién escribe decide; en el caso de la tildación diacrítica se puede requerir un él pronombre o un el artículo, y la computadora «no sabría» por cuál optar. Así podríamos presentar muchos ejemplos que señalan que el redactor es el único capaz de precisar el significado que quiere dar a las palabras en un texto (Portilla, 2009, pág 34).

Por otra parte Prisciliana (2007), explica, que la tildación general es uno de los temas más sencillos que se desarrollan dentro del aspecto normativo de la lengua, pero lamentablemente no es abordado adecuadamente por los profesores, ya sea porque muchos no tienen los conceptos claros o porque no realizan el reforzamiento necesario, a través de la práctica correspondiente. Trataremos aquí, de hacer llegar algunos alcances que pueden complementar la información previa que tienen los alumnos, con lo cual se pretende estimular el surgimiento de preguntas que permitan esclarecer las dudas en los discentes. (p.3).

Por tal razón, el Ministerio de Educación (MINED, 2009), expone que donde se falla, es en la práctica de estrategias que motivan la participación activa de los estudiantes en la expresión escrita por tanto, la escuela por ser la encargada de la formación de los estudiantes, debe implementar estrategias que garanticen la enseñanza de la escritura (p.34).

Las palabras compuestas, si bien se someten a las reglas de tildación general, constituyen un aspecto que debe ser tratado después de que el estudiante haya interiorizado los pasos de la tildación general. Hablar aquí de las palabras "sobreesdrújulas", que no son otra cosa que palabras compuestas con pronombres enclíticos, constituye un error que se arrastra desde décadas y que conviene erradicar de una vez por todas, pues su análisis corresponde a la tildación de palabras compuestas, su estructura (descripción morfológica; Lazo, 2010, pág 210).

Según Leyva (2001), en su teoría expone, que iniciar el proceso de enseñanza aprendizaje, planificando luego de una prueba diagnóstica, ayuda al docente a seleccionar el material adecuado para el grupo de que se trate. Leyva refiere que el material debe contener información ya conocida e introducir paulatinamente la novedosa. Las palabras desconocidas, deben ser buscadas en el diccionario y dar ejemplos sobre situaciones concretas, como presentar imágenes a través de láminas o películas, sobre todo en los primeros años de la escolaridad. Seleccionar las ideas principales, confeccionar con ellas un resumen, y luego una síntesis es un primer paso.

Las pruebas objetivas de verdadero y falso con justificación, ayudan a la reflexión crítica, lo mismo que un texto que contenga errores que los estudiantes deben descubrir. Los trabajos grupales, con opiniones personales y del conjunto y la discusión de ideas, son una estrategia a tener muy en cuenta (Fiszer, 2007, pág 49). 
Balmaceda (2001), afirma que la enseñanza de la Lengua Española, ocupa un lugar destacado en el conjunto de asignaturas de la Educación Primaria y en particular, en el primer ciclo. Su objeto de estudio es el propio idioma, nuestra lengua materna, fundamental medio de comunicación y elemento esencial de la nacionalidad (p.103).

De acuerdo a Lazo 2010, nuestro idioma, como todas las lenguas, está formado básicamente por sonidos o fonemas. En español, estos fonemas son veinticuatro. Las letras son la representación gráfica de estos sonidos. El alfabeto o conjunto de letras español consta de veintinueve unidades según la Academia Española de la lengua. Queda claro entonces, el origen del problema ortográfico en español es que hay más letras que sonidos o fonemas. Como indagadores en el problema estamos seguros de que también esto afecta en la aplicabilidad de las reglas generales de acentuación por la falta del uso del método fonético, analítico y sintético como eje principal para la buena acentuación de las palabras.

El autor Urrian (1985), señala en su postulación en el proceso educativo, debe realizarse a través de las siguientes estrategias: análisis de la palabra oral para conseguir una correcta discriminación auditiva del acento de intensidad, del hiato y del diptongo, evitando toda interferencia del fenómeno acústico con su representación gráfica. Formación de patrones acústicos de los fenómenos discriminados, tan vívidos que permitan su reconocimiento de manera global e instantánea. Formación de patrones visuales y motrices que permitan tildar automáticamente las palabras en forma correcta.

Carratala (2008), señala que la fórmula para la enseñanza aprendizaje de la ortografía acentual es: audición + recuerdo visual + sensación = a escritura correcta. Este proceso es el que debemos enseñar a nuestros alumnos antes de abordar sistemáticamente, el estudio de la temática de la ortografía acentual.

Una persona con buena estrategia ortográfica guarda la imagen de la palabra para el futuro, finalmente escriben las palabras que tienen almacenada en su mente y han reconocido con plena seguridad (carraala, 2008, p. 8).

\section{Materiales y métodos}

La investigación, se efectuó en el centro escolar "Isabel Amador", comunidad Dipina central de la Ciudad de Waslala, Región Autónoma Costa Caribe Norte (RACCN). Se realizó un diagnóstico a estudiantes, con el fin de indagar la problemática más sentida en el ambiente áulico, se aplicaron instrumentos para la práctica de estrategias metodológicas, en la búsqueda de soluciones mediante un aprendizaje significativo. Por sus características, es una Investigación - Acción-Participativa, del paradigma cualitativo, en la que se abordan las reglas generales de acentuación en octavo grado de Educación Secundaria a distancia. La población, la constituyeron 40 aprendientes 
de octavo grado y 2 docentes investigadoras, en acción directa con el estudiantado para un total de 42 personas. La muestra la representan 20 estudiantes, lo que significa el 50\% de la población en estudio. Las técnicas de investigación que se utilizaron: muestreo de lectura, entrevistas a estudiantes, docentes, padres y madres de familia, aplicación de exámenes y test a estudiantes, estrategias como: análisis y sopas de palabras, pastel y círculos de estudios participativos.

\section{Resultados y discusión}

Los componentes que inciden en esta temática: falta de práctica en las reglas generales de acentuación por ausencia de materiales didácticos como: libros de textos, guías de estrategias metodológicas y actividades lúdicas, pocas estrategias implementadas por docentes, desinterés de los estudiantes, el uso no responsable de la tecnología y débil hábito de lectura. Problemática que incide en la aplicación de reglas generales de acentuación en octavo grado de educación a distancia centro "Isabel Amador".

La situación se presenta, debido al desinterés hacia los estudiantes, desde los primeros niveles de Educación Primaria hasta los niveles superiores. Otro factor que interviene en el aprendizaje de los estudiantes es la falta de interés de los mismos, motivación por parte de la comunidad educativa, los juegos deportivos, y el trabajo de campo, todo esto afecta al estudiante en cuanto a su aprendizaje durante el transcurso del año lectivo.

En la práctica investigativa según las características de esta en particular, que consiste en acción participación, se inicia con la aplicación de un test como estrategia diagnóstica a los estudiantes. Los resultados se reflejan en la figura no. 1. En él, los datos cuantitativos, evidencian que la población estudiantil, presenta dificultades en todo los ítems que se evaluaron, sobre: conceptualización de palabras agudas, graves, esdrújulas y sobreesdrújulas.

Además, presentaron deficiencias en la acentuación de palabras, que se comprobó mediante la práctica de lecturas y en la misma, la clasificación de las palabras según el caso. En sentido general el $90 \%$ de la muestra, sujeto de la investigación, no tiene dominio en el uso de las reglas de acentuación, lo que se considera, como punto de preocupación y la necesidad de provocar cambios en esta situación.

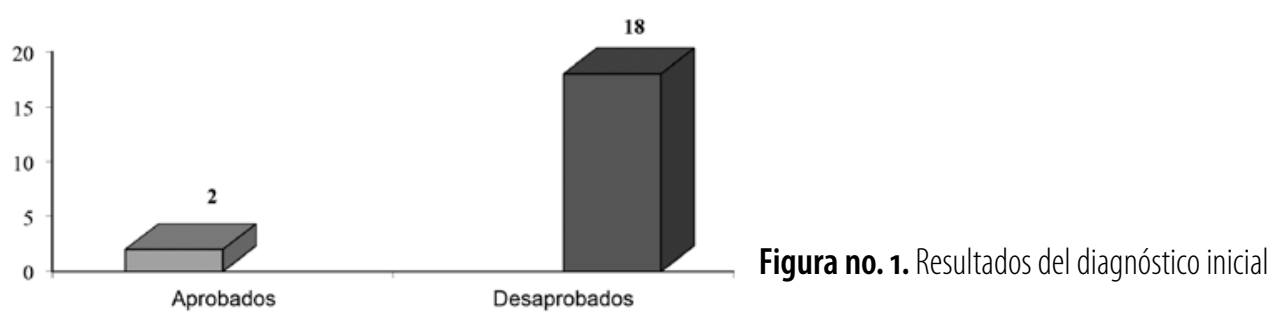


La figura no. 2. Exterioriza el comportamiento de aprobados y desaprobados, el diagnóstico por criterios de evaluación. En la misma, se destaca una problemática alarmante en el $75 \%$ de los parámetros: (identificación de palabras agudas, graves y en redacción).

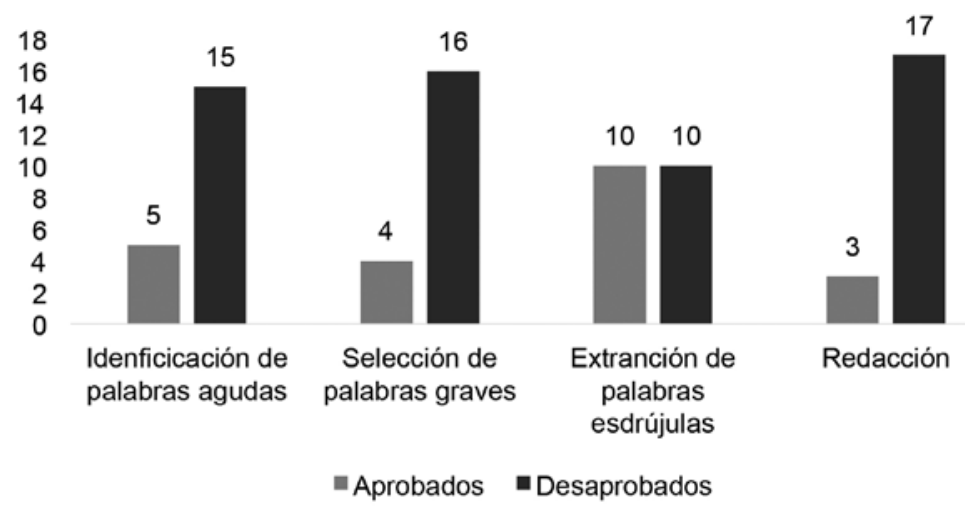

Figura no. 2. Resultados del diagnóstico por criterios de evaluación

Según el diagnóstico, este revela una serie de factores predisponentes que deja claro la cuota de responsabilidad por parte de cada uno de los involucrados en el proceso educativo. En el caso de los estudiantes, se verifica la falta de interés y hábitos de lecto-escritura y en los educadores: se relacionan con la dificultad de la aplicación de estrategias metodológicas creativas y eficaces, que despierten la motivación e interés; además que provoquen la participación activa en el proceso educativo para que este sea significativo, sin dejar de tomar en cuenta, la falta de materiales didácticos concretos, como apoyo en la construcción de un nuevo conocimiento, en el que se articulen la información previa de los educandos, con la que reciben en el proceso de enseñanza áulica.

\section{Diseño de un plan que facilite aplicar reglas generales de acentuación}

Después de la aplicación de la primer estrategia planteada en el plan de acción, realizamos la primera evaluación intermedia, luego se llevó a cabo un monitoreo continuo en la clase de Lengua y Literatura, para constatar el nivel de aceptación y asimilación de las otras estrategias implementadas en el centro educativo "Isabel Amador", para verificar el nivel de captación y dominio de las reglas generales de acentuación, se estableció coordinación entre docente y director del centro educativo para realizar las aplicaciones de las estrategias al final se realizó una evaluación final para obtener de los estudiantes un resultado concreto con respecto a nuestro tema. En esta investigación trabajamos con 20 estudiantes de $8^{\circ}$ grado de Educación Secundaria a distancia, a través de la observación directa, aplicamos diversas estrategias a los estudiantes. 
La figura no. 3. Muestra los resultados que se obtuvieron, después de la puesta en práctica de diferentes estrategias metodológicas con los estudiantes, en el mismo se observa una situación contraria totalmente, a la inicial, lo que significa el logro de los objetivo, aunque no en un ciento por ciento, sin embargo es significativo sobre todo, porque se verifican cambios sustanciales en el proceso de Investigación - Acción - Participativa.

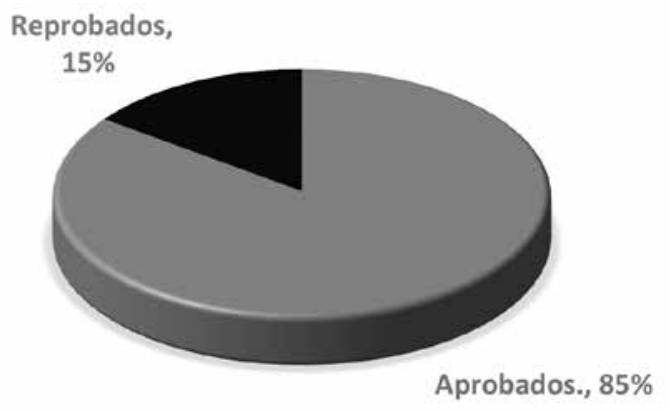

Figura no. 3. Resultados generales finales.

En la figura no. 4. Aparecen cuantitativamente, los resultados finales por criterios, en el que se caracteriza a los estudiantes con y sin habilidades, en relación a estos, los escolares desarrollaron habilidades en: conceptualización, clasificación de palabras, identificación de sustantivos y adjetivos, también la redacción de oraciones y párrafos.

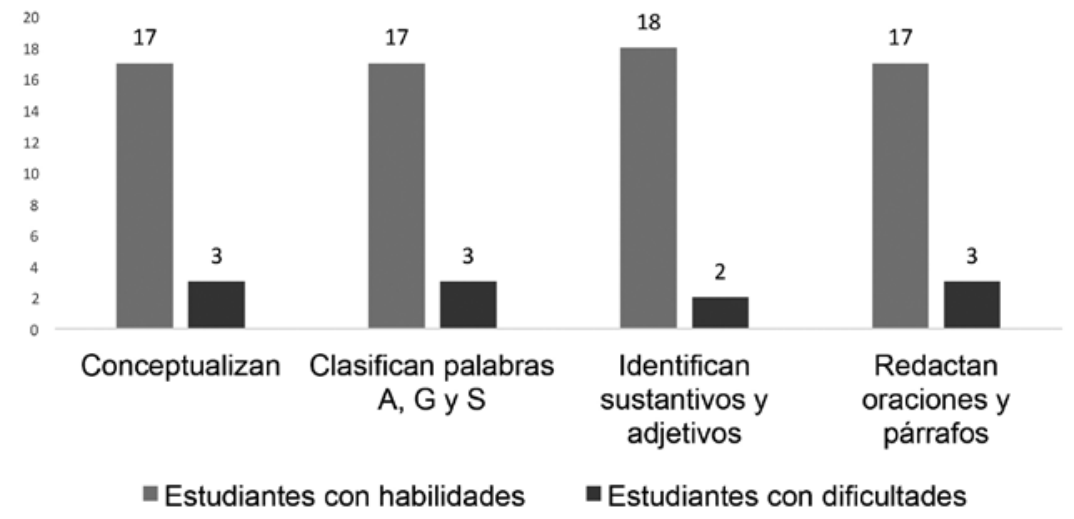
A) agudas
G) graves
S) sobresdrújulas

Figura no. 4. Resultados finales por criterios de instrumentos

De la misma forma, es meritorio hacer énfasis en relación a los estudiantes que no lograron desarrollar las habilidades con efectividad, pero se tiene la certeza que sí, mejoraron sus conocimientos que posteriormente, les servirán en los siguientes niveles académicos. 


\section{Conclusiones}

El objetivo fue el diagnóstico de la problemática de aplicación de reglas generales de acentuación en octavo grado, se presentan dificultades en cuanto al uso y manejo de las reglas generales de acentuación, tanto oral como escrita, y en otros aspectos a los que se les dio seguimiento, a través de las estrategias que se implementaron en el transcurso de la investigación.

La comunidad educativa, practica una integración positiva al fomentar nuevas estrategias didácticas, dinámicas lúdicas que permiten que los estudiantes se mantengan activos y participativos durante la clase. El diseño de un plan de acción con sus respectivas estrategias y aplicación objetiva facilitaron la enseñanza de las reglas generales de acentuación, la conceptualización, identificación, clasificación, acentuación, también a la mejora de la dicción correcta de palabras y lecturas, lo que permitió que los docentes como emisores, aporten una comunicación significativa y a los receptores un mensaje claro.

De las diferentes estrategias que se utilizaron, las más efectivas para el uso de las reglas generales de acentuación fueron: análisis de palabras, sopas de palabras y círculos estudios, estas ayudaron positivamente para que los estudiantes determinaran y clasificaran con facilidad palabras agudas, graves, esdrújulas, y sobre-esdrújulas, analizando los sonidos fonológicos, redacción de oraciones y párrafos sencillos con un lenguaje propio del contexto, que le proporcionó habilidades en su desarrollo cognitivo tales como: manejo de la ortografía, caligrafía, expresión oral y escrita, identificación de adjetivos y sustantivos, así como el uso del punto.

A través de la implementación de las diversas estrategias en estudio: se logra desarrollar la integración, interacción participativa entre los estudiantes, clases dinámicas y motivadoras, lo que genera la habilidad intelectual en la redacción de párrafos, palabras, dicción y acentuación, mediante el uso de las diferentes estrategias.

Las estrategias se basaron en el análisis de palabras, clasificación, redacción de textos y oraciones, se aplicó el método fonológico y dinámicas lúdicas relacionadas con el tema, estas actividades permitieron en los estudiantes la integración y participación activa-participativa, así como la memorización de reglas generales de acentuación.

\section{Recomendaciones}

En sentido general, que se practiquen y apliquen las reglas generales de acentuación tanto oral como escrita de forma sistémica y sistemática, por parte de los educadores y educandos. 


\section{A los docentes:}

Proponerse metas y pasos concretos en su quehacer educativo para el logro de los objetivos, mediante el uso de estrategias metodológicas adecuadas creativas e innovadoras, paralelamente crear ambientes atrayentes y lúdicos que motiven en los estudiantes la integración activa en los procesos de aprendizaje.

- Promuevan y desarrollen, procesos de intercambios entre docentes del mismo núcleo educativo, padres y madres de familia para una formación compartida.

- Promover el hábito de realizar círculos de estudio con estudiantes monitores y docentes, además los planes de reforzamiento de auto estudios.

- Que realicen un proceso de sensibilización a padres y madres de familias, destacando la importancia del acompañamiento de sus hijos e hijas para reforzar el aprendizaje del uso de las reglas de acentuación.

\section{A directores/as y asesores pedagógicos:}

Garantizar el seguimiento y monitoreo a los procesos de enseñanza - aprendizaje que se relacionan con el fortalecimiento en el uso adecuado de las reglas de acentuación

\section{Al MINED:}

Garantice el seguimiento a los centros de educación de secundarias a distancia, para concretar el proceso de enseñanza en la temática que se aborda, de la misma forma, que dote a estos centros y en sentido general de materiales didácticos como apoyo a la enseñanza de la asignatura Lengua y Literatura.

\section{Lista de referencias}

Balmaceda, O. (2001). Reglas generales de acentuacion. Belice: Monografias. com.

Calderon, F. (2010). La guia de educación. Honduras. Hinojosa.

Carratala. (2008). Técnicas para mejorar la ortografia. Madrid. ari64.

Castell, B. (2014). Ortografia acentual. Chile: chile.

Fiszer, D. (2007). Estraegias para el aprendizaje. Nicaragua. Ubemse.

Fuentes. (2010). Ortografia acentual. Madrid: shm.

Lazo, m. (2010). Nuestra Ortografia. Managua Nicaragua. Larousse. 


\section{EDUCACIÓN}

Leyva, C. (2001). Aulas libres. México.

MINED. (2009). Enseñanza de la lengua española. Managua, MINED.

Moreno, R. (2005). Monografia orografia 2. Nicaragua, MINED.

Portilla, D. (2009). Esudio Lexico. Madrid: redid. org.

Prisciliana, L. (2007). Niveles lexicos. Colombia: tesis. hsm.

Urrian. (1985). Preparandonos para una formacion mejor. México. 